\title{
Polydopamine as a stable and functional nanomaterial
}

\author{
Salima El Yakhlifi ${ }^{1,2}$ and Vincent Ball ${ }^{1,2,{ }^{*}}$
}

1: Université de Strasbourg

Faculté de Chirurgie Dentaire

8 rue Sainte Elisabeth

67000 Strasbourg

France

2: Institut National de la Santé et de la Recherche Médicale

Unité mixte de recherche 1121

11 rue Humann

67085 Strasbourg Cedex

France

*Corresponding author: vball@unistra.fr

\begin{abstract}
The mussel inspired chemistry of dopamine leading to versatile coatings on the surface of all kinds of materials in a one pot process was considered as the unique aspect of catecholamine for a long time. Only recently, research has been undertaken to valorize the simultaneous oxidation and colloid formation in dopamine solutions in the presence of an oxidant. This mini review summarizes the synthesis methods allowing to get controlled nanomaterials, either nanoparticles, hollow capsules or nanotubes and even chiral nanomaterials from dopamine solutions. Finally the applications of those nanomaterials will be described.
\end{abstract}

Keywords: polydopamine, templates, biomimetic approaches, nanomaterials.

6849 words

0 Tables

8 Figures 


\section{Introduction}

Surface modifications play a central role in a variety of application domains from electronics to medicine. There are multitude of techniques for chemical modification and surface functionalization which are described in a large number of review articles [1]. The current coating toolkits include irradiative chemisorption, layer-by-layer process [2] (LbL), self-assembled-monolayers (SAM) [3], chemical vapor deposition (CVD)... However, most of these methods have limitations in that they are substrate specific, some of them require complex multistep processes and others need expensive equipment. Hence the need to search for easier and universal coating processes is important.

From this point of view, Nature is an endless source of inspiration. Indeed, many biological systems have developed efficient solutions to adhere to different types of surfaces through a combination of different interactions. Thus, several universal coatings have been inspired by a variety of biological systems: gecko [4], blood proteins from the coagulation cascade [5], snail [6]... For example, phenolic biomolecules that are present in tea, chocolate, and wine and many other plants, have been identified as an interesting approach for the formation of colorless multifunctional coatings [7].

Similarly, polydopamine (PDA), known for its ability to strongly attach to almost all kinds of substrates, was inspired by mussels' foot proteins ( $\mathrm{mfp}$ ). Introduced in 2007 by Messersmith, Lee et al. [8, 9], PDA rapidly became one of the most popular coating methods. For instance, it has been shown that PDA can modify Teflon microchannel walls and thus lead to some applications of the modified chips that are difficult with native Teflon chips [10]. The same holds true to make Teflon cell adhesive [11].

Owing to the presence of catechol (as in L-Dopa) and amine (as in L-Lysine) in mussels foot proteins, the dopamine molecule, which contains these two latter groups, has been identified as a potential and efficient precursor of universal coatings that can be functionalized at will. Indeed, functional molecules containing nucleophilic groups (thiols, amines) can be easily immobilized onto quinones present in the structure of PDA to obtain synthetic derivatives [12-14]. 
Despite extensive investigations over the past decade, the chemical basis of the adhesion properties of PDA, containing catechol and amino groups, is still not totally understood. Even if the simultaneous presence of L-Lysine and L-DOPA in mfp proteins has been demonstrated to allow for strong adhesion on a vast repertoire of substrates [15, 16], such an accurate knowledge of the adhesion mechanism is not yet available for PDA. However, it is accepted that PDA adhesion is a complex process based on film deposition and growth in which primary amine groups, besides catechol moieties, play a huge role. Indeed, it was reported that replacing the amine group of dopamine with a hydroxyl group results in the formation of polymeric materials completely devoid of adhesion and film forming properties [18] but the addition to amines in a caffeic acid solution allows for the deposition of a film at the solid-liquid interface [19]. Additionally, catechols are anchors that immobilize the polymer coating onto a surface and allow to form intermolecular chemical crosslinks [20] improving the stability of the obtained coatings.

Due to its easy processing, in a one pot preparation method, and its chemical versatility, PDA proved to be a powerful tool for chemical and biomedical applications [20, 21]. Important efforts have hence been done to better understand and to control the formation of PDA. This appeared however as a challenging task owing to the structural heterogeneity of this material. PDA is either considered as a polymeric material or a self-assembled material made from oligomers of 5,6dihydroxyindole [22-24], the final oxidation product of dopamine (Fig. 1). Recently some evidence for the role of cation $-\pi$ interactions in the formation and structure of PDA has been provided [25].

Unfortunately the oxidation process leading to PDA coatings from solution processes, oxidation and polymerization/self-assembly, leads also to the formation of a useless precipitate from the solution. This is a major drawback owing to the high cost of catecholamines. Efforts have then be devoted to avoid such a precipitation process and to control the self-assembly of the oxidation products of dopamine (and other catecholamines) in solution to get stable nano-colloids. The obtained PDA nanoparticles and other nanomaterials have potential as photothermal agents and can be used to modulate near infra-red (NIR) responsive functional nanomaterials. All these properties make PDA a material of choice for a broad range of applications. Therefore, it is necessary to control the structure and the properties of polydopamine to exploit it efficiently. 
In this review, we summarize the features of current strategies to develop controlledsize and stable polydopamine nanomaterials, which include the use of surfactants, polyelectrolytes, proteins and other organic molecules like folic acid. Also, some applications of polydopamine based-nanomaterials will be described. It is not our aim herein to review the application of PDA coatings on nanoparticles, a field that has already been covered previously [26].

The present minireview is organized in the following manner: we will first describe the analogies between PDA and eumelanin as an inspiration source to produce nanosized PDA. This analogy will be the basis to control the self-assembly process of PDA in a biomimetic way during the oxidation of dopamine in controlled nanostructures. Whose nanostructures have photothermal and other properties common with eumelanin and offer a plethora of possible applications in biomaterials science in particular and in materials science in general.

\section{Polydopamine as a eumelanin analogue}

There are two major pigments in the skin: eumelanin (black/brown) and pheomelanin (reddish/brown). Each is produced in melanocytes and derives from the amino acid L-Tyrosine which is hydroxylated and cyclized through the action of Tyrosinase and other pigmental enzymes [27]. This latter enzymes play a role in converting Tyrosine to L-DOPA and then L-DOPA to the corresponding quinone (Fig. 1) [28, 29]. 

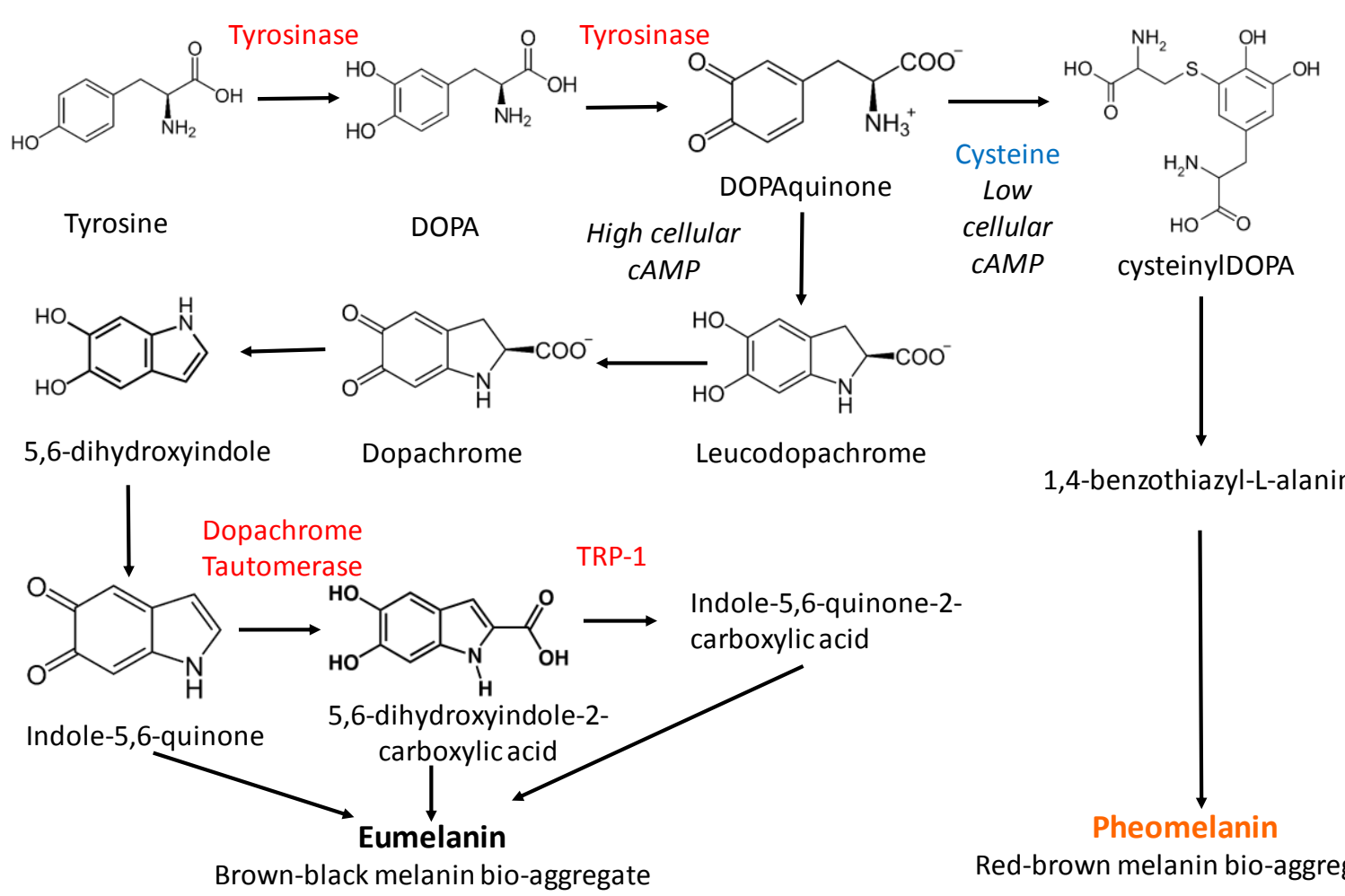

1,4-benzothiazyl-L-alanine

Brown-black melanin bio-aggregate

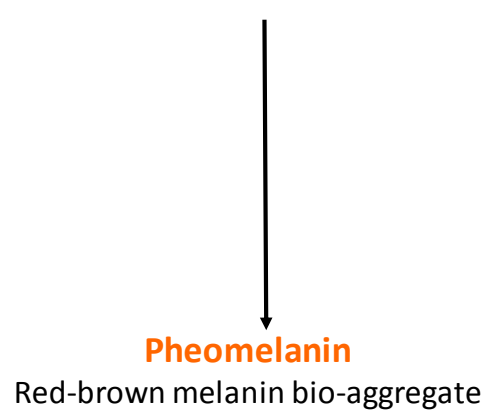

Fig. 1: Chemical pathways leading to eumelanin and to pheomelanin and the enzymes (in red characters) implied in the different chemical steps. Modified from ref. [27] with authorization. Nomenclature: DOPA: 3,4-dihydroxyphenylanaline, TRP1: Tyrosinase related protein 1.

Owing to a similar oxidation pathway, PDA shares various physicochemical properties with eumelanin. The building blocks of eumelanin are 5,6-dihydroxyindole2-carboxylic acid (DHICA) and 5,6-dihydroxyindole (DHI) (Fig. 1) [30]. Eumelanin and PDA have extremely close absorption spectra covering the entire wavelength range of the UV-vis domain in the electromagnetic spectrum [28, 31-33]. In addition, their fluorescence quantum yield is extremely low with most of the absorbed light being converted into heat [34]. This is well known in the skin which undergoes heating after exposure to sunlight. Concerning PDA, this property is at the basis of applications as a possible photothermal material.

Therefore, PDA is also referred to as a "eumelanin-like" material since DHI (and its oxidized forms) is the key building block of both PDA and eumelanin [28]. Actually, the term of polydopamine is misleading since it is probably not a real polymer but more likely a complex self-assembled amorphous material [24]. Based on ab initio 
calculations of the structure-property-function relationship of PDA and eumelanin, a large number of oxidized $\mathrm{DHI}$ oligomers have been evaluated to compare their molecular structures. The obtained findings highlighted that more planar structures are energetically more favorable and that cyclic molecular structures could reduce the energy of a DHI based tetramer and make it more stable. This afforded a set of molecular models for more specific modeling of eumelanin-like materials [35]. This assumption of tetrameric dopamine based structures [36-38] as building blocks of PDA is however heavily debated [39] and additional experimental proofs are required.

Owing to their similar chemical composition, PDA as a "synthetic eumelanin" are the focus of increasing interest as UV-absorbing agents, antioxidants and free radical scavengers. Those properties are particularly interesting in the colloidal state, provided the oxidation products of catecholamines may be stabilized against aggregation and/or flocculation. In addition eumelanin in the skin is always of controlled hierarchical size and surrounded by proteins [40]. This is not the case of PDA which is an amorphous precipitate when produced in the absence of additives. It is hence a natural idea to added well suited additives to dopamine to control its self-assembly/polymerization process.

\section{How to avoid precipitation in solution and to produce stable nanomaterials}

Autoxidation of dopamine in alkaline aqueous solutions is a standard method for PDA formation. But unlike as for PDA coatings, the preparation procedures of PDA nanoparticles (PDA@NPs) are still limited and new protocols are widely desirable. Indeed, some properties, as photothermal and free radical scavenging, can be influenced by the PDA@NPs size owing to obvious surface /volume ratios. Synthetic eumelanins yield huge polydisperse aggregates [41]. However, if the catecholamine concentration is extremely low, the regime where small and stable nanoparticles are formed in the absence of film deposition on the walls of the reaction beaker can be reached. The colloidal stability of those nanoparticles is due to their $\mathrm{pH}$ dependent zeta potential: the PDA film and the obtained particles are negatively charged above the isoelectric point of PDA which is close to 4.9 [42]. An interesting way to improve the surface charge on water soluble melanin was to perform their synthesis in the presence of a higher partial pressure in oxygen 
allowing to increase the number of carboxylic groups on the obtained colloids [43]. Grafting of thiol-terminated methoxy-poly(ethylene glycol) on polydopamine synthesized in the presence of $\mathrm{NaOH}$ allowed to produce stable and biocompatible nanoparticles with less than $100 \mathrm{~nm}$ diameter [44].

At higher concentration in dissolved catecholamines, which is required to obtain a better yield in nanoparticles, the obtained colloids are too large to be stabilized and precipitation occurs. Fortunately, It has been shown that the addition of additives in the dopamine solution at typically $1 \mathrm{mg} \cdot \mathrm{mL}^{-1}$ (or $5 \mathrm{mM}$ ) like surfactants, polymers, polyelectrolytes or some proteins allow to get PDA of controlled size. Polymers like poly(vinyl alcohol) [45] and surfactant micelles [46] allow for the production of stable suspensions of PDA. Strikingly, in the presence of sodium dodecyl sulfate or cetyltrimethylammonium bromide, the hydrodynamic diameter of the PDA particles formed in the presence of oxygen at $\mathrm{pH}=8.5$ decreases with an increase in surfactant concentration up to the critical micellar concentration $(\mathrm{CMC})$. For surfactant concentrations higher than the $\mathrm{CMC}$ the diameter of the PDA containing particles is similar to that of the surfactant micelles (Fig. 2), suggesting that the PDA formation occurs preferentially in the core of the micelles. This point requires of course additional investigations.

A

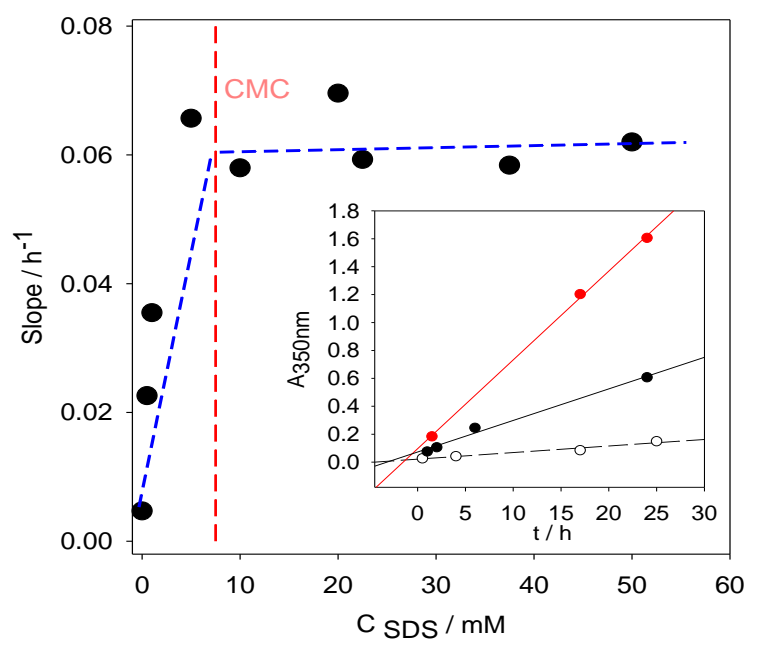

B

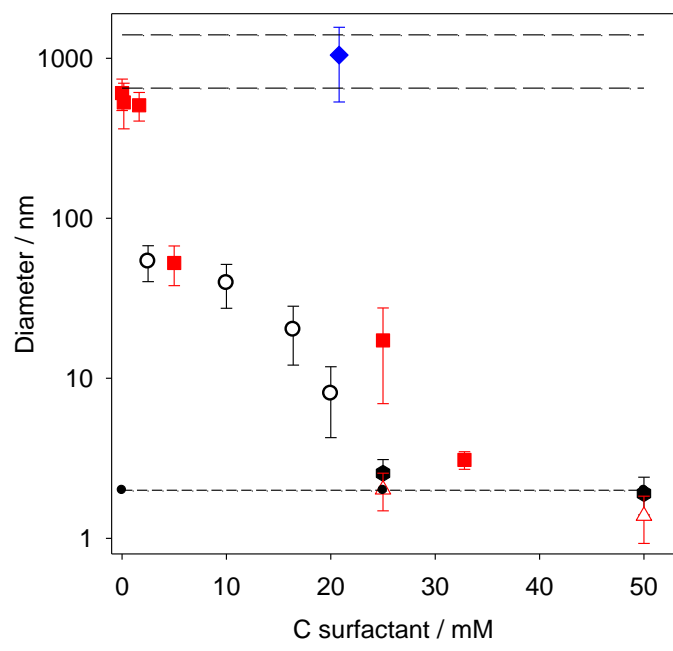

Fig. 2 : A : Rate of PDA formation as followed by UV-vis spectroscopy at $\lambda=350$ $\mathrm{nm}$ as a function of the concentration in SDS (-----). Each point is obtained from a curve as those represented in the inset (SDS at $0(O) 0.5(\bullet)$ and $\bullet 5$ $m M)$. The straight lines correspond to linear regressions to the data. 
$B$ : Evolution of the size of "polydopamine" aggregates as a function of the surfactant concentration in the case of SDS (O), CTAB ( $\square$ ) and Triton X-100 $(\forall)$. The upper long dashed lines correspond to the size domain of "polydopamine" prepared in the absence of surfactant whereas the lower short dashed line corresponds to the size of the surfactant micelles (measured in the case of SDS ( $\mathbf{\square})$ and CTAB ( $\triangle)$ ).

Modified from ref. [46] with authorization.

Another method to control PDA formation and to avoid precipitation is the use of boric acid as an adjuvant that is able to stop the deposition of PDA on surfaces and simultaneously to control self-assembly of PDA in solution to get stable colloidal aggregates [47]. Boric acid forms strong hydrogen bonds with catechol moieties (Fig. 3B) which has the consequence to strongly increase the oxidation potential of dopamine (Fig 3A). Adding boric acid to dopamine solutions after a given oxidation and self-assembly duration allows to stop the growth of the PDA based nanoparticles to a controlled and reproducible size provided the boric acid/dopamine ratio is higher than 5 [47]. 
A

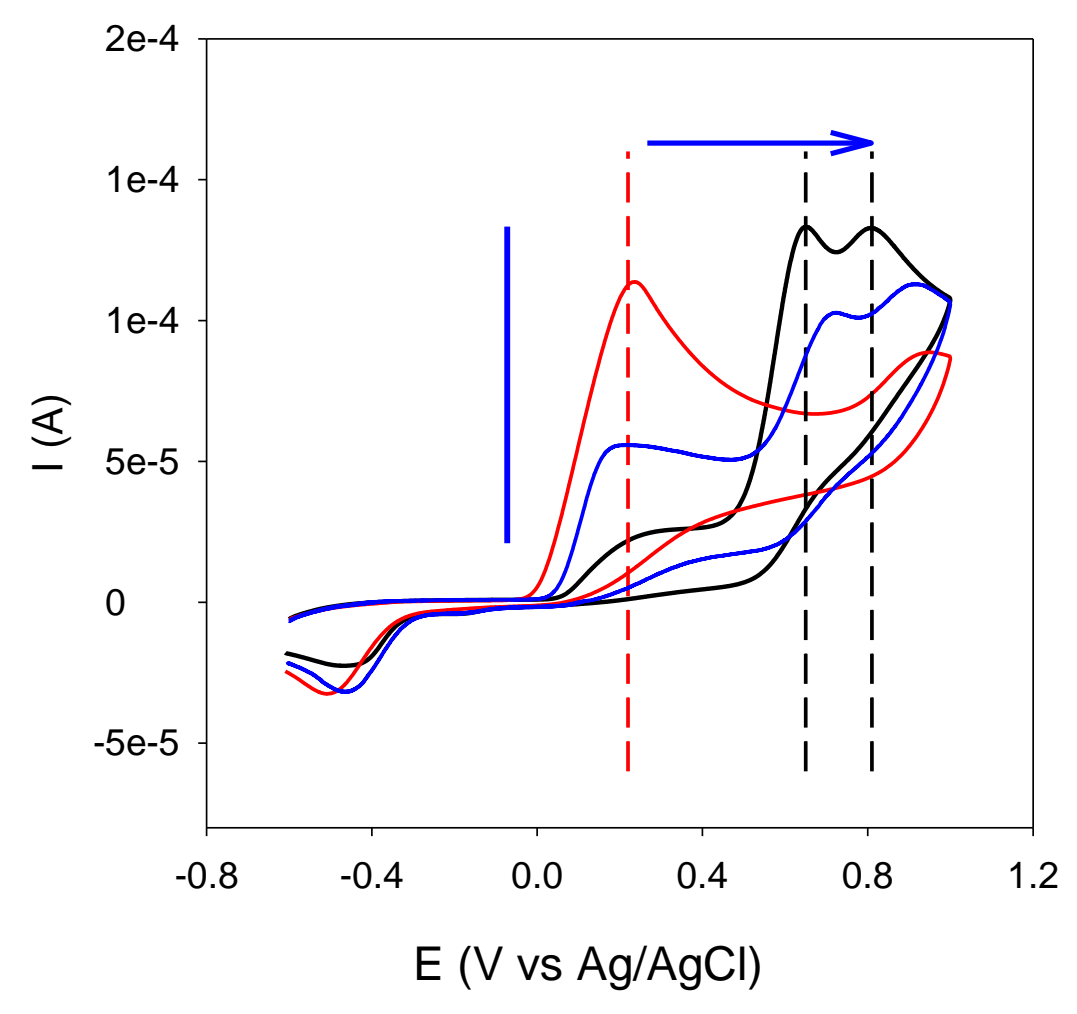

B<smiles>NCCc1ccc(O)c(O)c1</smiles>

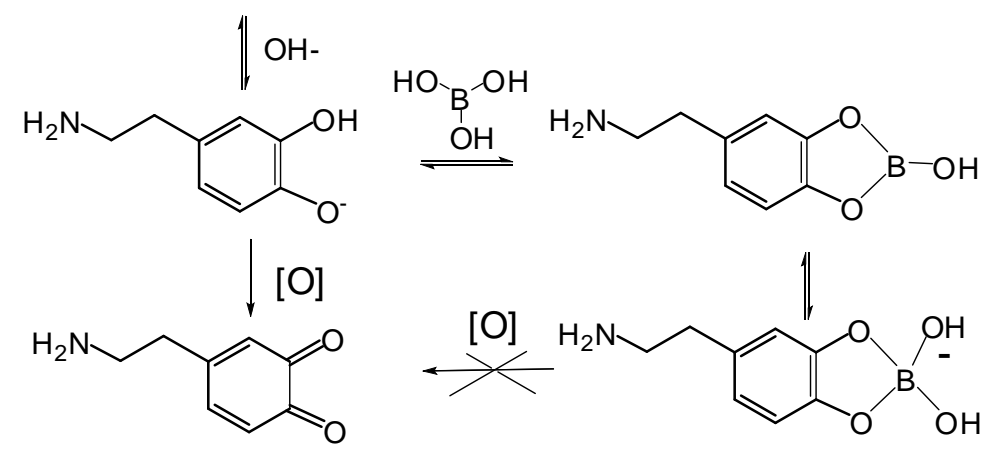

Fig. 3: Interaction of boric acid with dopamine and its influence on the electrochemical behavior of dopamine. A: Cyclic voltammetry of dopamine dissolved at $3.5 \mathrm{mM}$ in Tris buffer at $\mathrm{pH}=8.5$ (red line) and in the same buffer but in the presence of $43 \mathrm{mM}$ (blue line) and $430 \mathrm{mM}$ (black line) boric acid. The blue arrows indicate the evolution of the cyclic voltammetry curves upon an increase in the boric acid concentration. B: Interaction of dopamine with boric acid and inhibitory effect of complex formation on oxidation to quinone and PDA formation. Modified from ref. [47] with authorization. 
A recent study showed that a vast repertoire of oxidants can be used to rapidly prepare polydopamine under aerobic or anaerobic conditions, also in mildly acidic aqueous solutions, and at room temperature [48]. Also, the use of strong oxidants such as sodium periodate leads to PDA formation in a much shorter time [49]. Another effort to control the size of the PDA@NPs consists in adding either strong free radical scavengers (i.e edaravone) or stable free radicals (i.e PTIO) [50] during the synthesis, both resulting in a decrease in the NPs size (Fig 4).

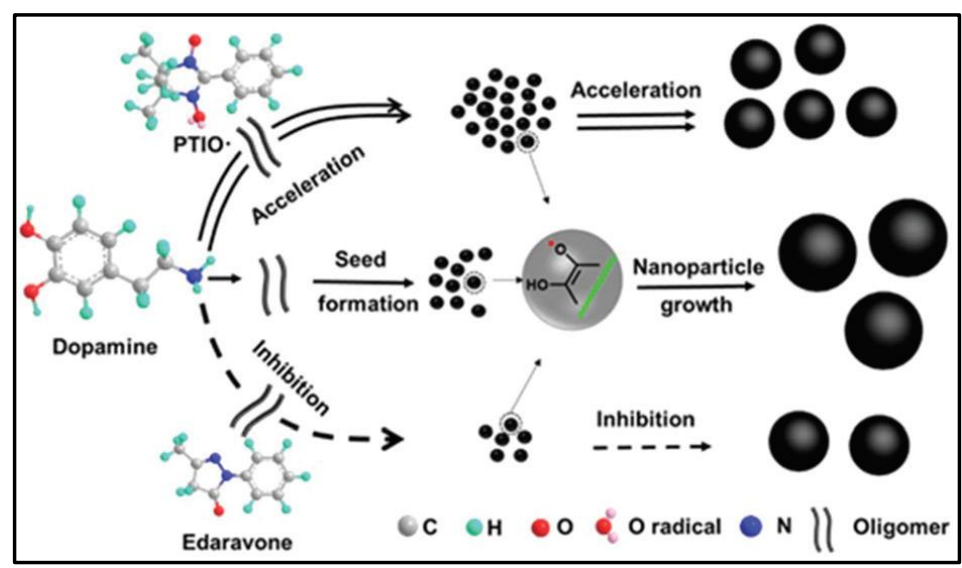

Fig.4: schematic illustration of the size controlled synthesis of PDA NPs by using radicalar species. Reproduced from ref.[50] with authorization.

Yet, the problem of the precipitation is still not totally resolved. The size of PDA@NPs can be modulated by changing the amount of dopamine monomers dissolved in a water in oil microemulsion to elaborate $\mathrm{pH}$-activatable nanoparticles with an average diameter from 25 to $43 \mathrm{~nm}$ [51].

Inspired by the fact that eumelanin is always surrounded by a layer of proteins [40], a recent study has shown that the whole pool of proteins from the egg white allows to produce well controlled and stable eumelanin like material from DHI [52]. However, the molecular origin of this controlled oxidation and self-assembly was not investigated, even if many purified enzymes like tyrosynase can play a similar role during the oxidation of L-DOPA $[53,54]$.

Inspired from the proteins present in the skin melanocytes, a specific diad of amino acids in proteins, L-lysine $(\mathrm{K})$ and L-glutamic acid $(\mathrm{E})$, was shown to allow for specific control in the oxidation-self-assembly of dopamine to obtain biocompatible polydopamine@protein nanoparticles. Experiments were performed with single model peptides GKEG, GKGEG and GKGGEG in which the K and E were directly 
adjacent to each other or separated with one or two Glycine residue. It appeared that $\mathrm{K}$ and $\mathrm{E}$ have to be adjacent in the amino acid sequence to exert a templating effect in the assembly of PDA particles [55].

Human Serum Albumin (HSA), also containing KE diads, was found to increase the rate of PDA formation from dopamine and to allow for the formation of stable, biocompatible nanoparticles [56]. Other proteins that also contain the KE sequence were found to play a similar role such as Alkaline Phosphatase from bovine intestinal mucosa (ALP) (Fig. 5) [57].

The oxidation of dopamine in the presence of ALP in acidic conditions and using sodium periodate as the oxidant allows to produce small nanoparticles in a much faster way than standard conditions using Tris buffer at $\mathrm{pH} 8.5$ and dissolved $\mathrm{O}_{2}$ as the oxidant. An increase in protein concentration induces a significant reduction in the average size of the PDA nanoparticles (Fig. 5) [57] and a simultaneous reduction of the deposition of PDA films on the walls of the reaction beakers. On the other hand, proteins devoid of KE diads like chicken egg white lysozyme seem neither to affect the size of PDA formed in its presence nor the PDA deposition on the walls of the reaction beaker.

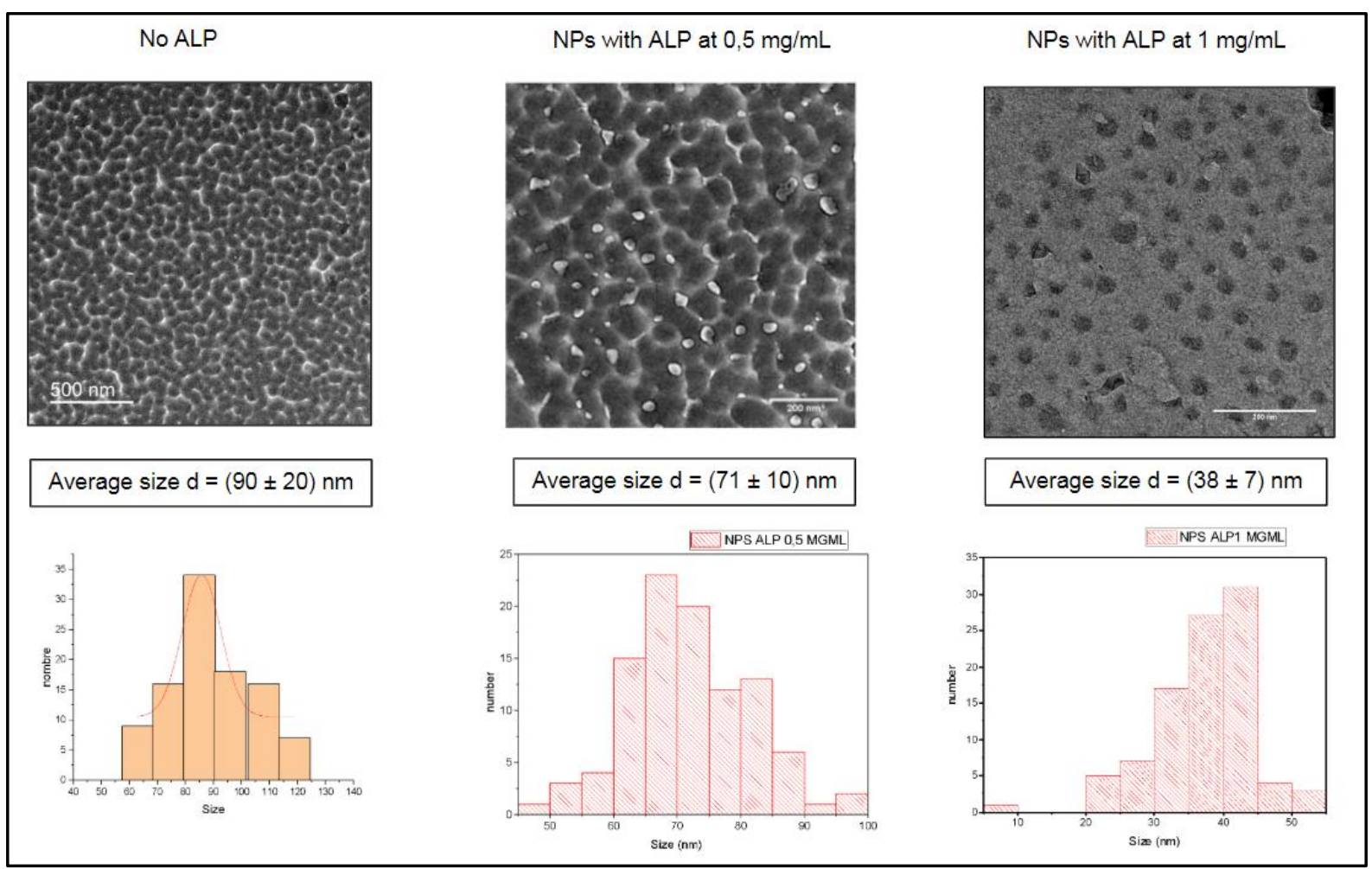


Fig. 5: Top: representative TEM micrographs of PDA@NPs produced after 6h of oxidation in the presence of $20 \mathrm{mM}$ sodium periodate from the dopamine solutions after the subsequent dialysis against Tris buffer, as a function of the added concentration in ALP. Bottom: size distribution of the particles obtained by analyzing 100 particles. Reproduced from ref. [57] with authorization.

The mechanism by which the two amino acids in the KE diad act to modify the oxidation and self-assembly pathways of dopamine has been investigated by means of molecular dynamics simulations. It was found that the residence time of unoxidized dopamine was increased on a KE dipeptide in comparison with other dipeptides due to hydrogen bond formation between the carboxylic group of LGlutamic acid with the catechol $\mathrm{OH}$ groups in synergy with a cation- $\pi$ interaction between the amino group of L-lysine and the aromatic cycle of the catechol [57].

The KE diad of amino acids plays a clear role in the formation of dopamine based nanomaterials but it is not unique in this effect since tripeptides made from Lphenylalanine, L-aspartic acid and L-Tyrosine also strongly affect the structure and the size of the oxidation product of L-tyrosine [58].

The PDA nanoparticles prepared in the presence of KE containing enzymes display the enzymatic activity expected for that enzyme, meaning that part of the enzyme present in the nanoparticle structure remains active and accessible to its substrate. However the fraction of active enzyme is not yet known as well as the enzyme distribution in the nanoparticles. There is no definitive proof that the obtained PDA@protein nanoparticles are of the core-shell type even if high resolution TEM images suggest such a structure [57].

Not only molecules like surfactants and peptides but also organic molecules like folic acid allow to direct the shape of PDA based nanostructures to nanofibers [59]. $\pi-\Pi$ interactions are responsible for such a size and morphology control [60]. Interestingly, when resveratrol-dopamine mixtures are oxidized by $\mathrm{O}_{2}$ in $5: 1$ water:ethanol mixtures, composite nanocapsules are obtained in the absence of templating agents [61].

\section{Sacrificial templates and polydopamine based nanomaterials}


Hollow capsules and nanotubes can be obtained by depositing polymers on templates having the shape of the desired nanomaterial followed by selective dissolution of the template. Hollow polyelectrolyte based nano and microcapsules could be obtained after the alternated adsorption of oppositely charged polyelectrolytes on polymer based or inorganic materials using the layer-by-layer (LbL) deposition method [2]. The LBL deposition method is however a multistep and time consuming process requiring the elimination of the non adsorbed polyelectrolytes, by centrifugation, after the completion of each adsorption step. Each adsorption step leads to a thickness increment of only a few nanometers, which is dependent on the ionic strength of the polyelectrolyte containing solution. Owing to the one step functionalization of surfaces with PDA, hollow capsules were first produced on non porous silica particles varying in diameter from 0.5 to $5.0 \mu \mathrm{m}$. By dissolving the silica core with $2 \mathrm{M}$ hydrofluoric acid $+8 \mathrm{M}$ ammonium fluoride, intact and hollow PDA capsules were obtained provided the oxidation time of dopamine was higher than $3 \mathrm{~h}$, leading to a minimal capsule wall thickness of $10 \mathrm{~nm}$, before etching of the silica core [62]. The concept was extended to porous silica templates in the same investigation. Using porous calcium carbonate templates multi-enzymatic systems were obtained using the same methodology [63]. Hollow, micrometer long nanotubes were obtained on curcumin [64] and $\mathrm{ZnO}$ templates from dopamine solutions [65].

\section{Applications of polydopamine based nanomaterials}

Due to high selectivity and minimal invasiveness, photothermal therapy is emerging as a powerful technique for cancer treatment. Photothermal agents and near-infrared laser exposure allow to target specific tumor sites. A photothermal agent based on dopamine-melanin colloidal nanospheres has been developed for cancer therapy. These nanoparticles have shown good photothermal conversion capability. It was demonstrated that a NIR laser irradiation at $2 \mathrm{~W} / \mathrm{cm}^{2}$ for 500 seconds of a PDA nanoparticles suspension can produce a significant temperature increase. Compared to pure water as negative control, the temperature of the PDA suspension increased by $33.6^{\circ} \mathrm{C}$ instead of $3.2^{\circ} \mathrm{C}$ for the water [66].

These results pave the way for eumelanin-like nanomaterials as efficient agent for cancer therapy. 
Indeed using the control exerted by KE containing proteins [55] on the self-assembly and size control of PDA, we managed to prepare transferin containing PDA based nanoparticles able to target melanoma cells, to be phagocytozed by them and able to kill them using the photothermal properties of PDA [67].

Using the antioxidant property of PDA [44], an investigation was made to study the use of PDA@NPs as scavengers of reactive oxygen species (ROS) in oxidative stress-induced periodontal disease. Interestingly PDA@NPs exhibited very good antioxidative performance [68]. The antioxidant properties of PDA based nanocolloids are conserved even in the presence of a poly(ethyleneoxide) based capping layer [44].

One of the primary biological role of eumelanin is the protection of the skin against UV-induced nuclear DNA damage. A lack of eumelanin production in humans can cause severe irreversible diseases. Thereby, novel synthetic routes to mimic eumelanin-like particles are widely expected. Human epidermal keratinocytes (HEKa), incubated with melanin-like particles, displayed significantly higher viability against UV irradiation than other groups including $\mathrm{SiO}_{2} @ P D A, P D A @ \mathrm{SiO}_{2}$, Au@NPs, all similar to melanin-like nanoparticles in size and surface charge (Fig.6) [69].

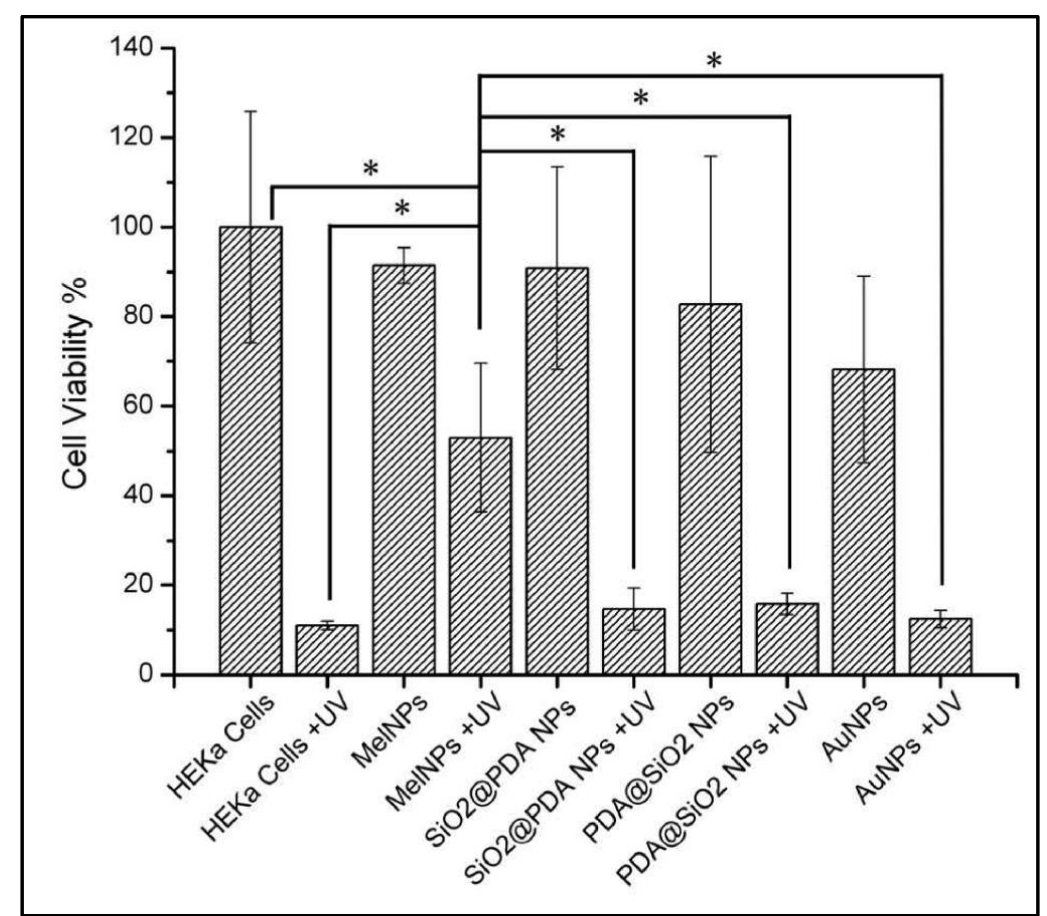

Fig.6: HEKa cell viability with and without UV following a 3 day incubation with melanin-like NPs (MeINPs), SiO ${ }_{2} @ P D A$ core-shell NPs,PDA@SiO 2 core-shell NPs, Au@NPs ${ }^{*} p<0.05$ 
Reproduced from ref. [69] with authorization.

Due to the combination of adhesive ability and redox activity, and their strong chelation for metal cations, PDA@NPs are promising candidate for applications in biomedicine and biodetection fields. For instance, they have been recently used as a support to load functional nanomaterials. In particular, Prussian Blue nanoparticles (PB@NPs) are good candidate as peroxidase mimics for electrochemical biosensors thanks to their excellent catalytic activity. They nevertheless have limitations: their aggregation in aqueous solution and their intrinsic color interference. An outcome consists of anchoring dispersed PB@NPs on the surface of PDA nanospheres. Thus resulting in a better dispersion in water and avoidance of the intrinsic color interference. Then the PDA/PB nanocomposites could trigger the color reaction of several substrates [70].

Because of the increase in bacterial resistance to many antibiotics it is an urgent challenge to develop new antibacterial materials. In this context, a multifunctional chitosan functionalized Chlorin e6 based magnetic polydopamine nanoparticles was constructed to fight methicillin-resistant Staphylococcus aureus (MRSA) infection by photodynamic therapy [71]. Magnetic PDA nanoparticles prepared by mixing ferric chloride hexahydrate and dopamine, were used to improve the MRSA cells enrichment performance.

Another group demonstrated the therapeutic efficiency of the combined photodynamic and photothermal therapy from Chlorin e6 and PDA under irradiation at $665 \mathrm{~nm}$ on bladder cancer cells [72]. In this investigation, PDA was used for its ability to encapsulate and load drugs but also for its photothermal properties.

When PDA is synthesized using $\mathrm{H}_{2} \mathrm{O}_{2}-\mathrm{Cu}^{+2}$ as the oxidant, the obtained nanoparticles are fluorescent and can be used as sensors for $\mathrm{Al}^{3+}[73]$. Formaldhyde sensing is possible with low interference from other gases like carbon dioxide, hydrogen sulfide methane, benzene and ammonia when hollow PDA nanotubes are fixed on the surface of a quartz microbalance based gravimetric transducer. The selectivity for formaldehyde originates from the interaction between the aldehyde with $-\mathrm{NH}$ - groups present on the surface of PDA [65]. The sensor was reversible for at least three exposures to gaseous formaldehyde at $30 \mathrm{ppm}$. The detection limit for formaldehyde was lower than $100 \mathrm{ppb}$ [65]. 
Nanometer sized PDA fluorescent nanoparticles can also be obtained through anodic microplasma electrochemistry [74]. This process allows to control the nanoparticles' size through a progressive acidification of the reaction medium to a $\mathrm{pH}$ value close to 5 where the spontaneous oxidation of dopamine is not possible anymore. Those nanoparticles have been shown to sensitive sensors for uranium cations, with a detection limit equal to $2.1 \mathrm{mg} \cdot \mathrm{L}^{-1}[74]$.

PDA based nanomaterials have been used for energy conversion processes and those applications have already been reviewed [75].

Owing to its strong absorption over the whole UV-vis part of the electromagnetic spectrum, PDA nanoparticles packed as colloidal crystals allow to absorb the scattered light which usually produces milky white colors in colloidal crystals. Centrifugation of PDA nanoparticles produces from the oxidation of dopamine in water/methanol mixtures (4/1 in volume proportion) allows to make films displaying structural colors [76] depending on the particles' diameter [77] as well on the nature of the monomer [78] used to produce the nanoparticles (either L-DOPA, dopamine or norepinephrine). Very recently, the diameter of PDA based nanoparticles and the structural color of PDA based photonic crystals has been modified by grafting poly(hydroxyethyl methacrylate) chains on PDA nanoparticles covered with an atom transfer radical polymerization (ATRP) initiator. Those PDA nanoparticles have been produced in a two step process first starting from dopamine in the presence of Tris buffer at $\mathrm{pH}=8.5$ (dissolved $\mathrm{O}_{2}$ being the oxidant) and following by the addition of 2bromoisobutyryl bromide $(\mathrm{BiBB})$ modified dopamine, to produce core shell nanoparticles around $220 \mathrm{~nm}$ in diameter, where the shell is made from BiBB-PDA acting as the ATRP initiator [79]. All this research relies on the fact that PDA is closely related to eumelanin which is the material used to produce the structural colors of the male peacocks' feathers [80].

Finally, chiral PDA nanoribbons were obtained by performing the oxidation of dopamine in the presence of templates made from self-assembled phenylalanine based amphiphiles. The racemic mixture of these two amphiphiles produced flat, achiral tapes after dopamine oxidation [81].

\section{Conclusions and perspectives}

Recent research has shown that PDA and similar compounds obtained through the oxidation of catecholamines appear not only to be mussel inspired 
coatings but also eumelanin inspired colloids and nanomaterials. Up to now proteins and molecules like folic acid appear to be the best candidates to allow for a fine control, protein or folic acid concentration dependent, of the nanoparticles shape and size. Some basic mechanistic aspects of the role of certain specific amino acid sequences (KE for instance) in the controlled oxidation and self-assembly of PDA have been acquired even if an intensive research effort is still required. It is remarkable that skin eumelanin is a hierarchical material also surrounded by proteins.

The enzyme containing PDA based nanoparticles display the enzymatic activity of the incorporated enzyme suggesting that at least some of them keep their conformation and are accessible to the water soluble substrates. The optical properties of the PDA based nanocolloids make them very promising photothermal materials in combination with the possibility to functionalize them with cell targeting molecules. Finally PDA interacting strongly with solutes like aldehydes and metallic cations, may be used as sensing platforms with high selectivity and specificity. Bioprinting of this biological ink, offering excellent biocompatibility, is also in plan for future applications.

\section{References}

[1] Q. Wei, R. Haag, Universal polymer coatings and their representative biomedical applications. Mater. Horiz., 2 (2015) 2, 567-577.

[2] J.J. Richarson, J. Cui, M. Bjornmalm, J.A. Braunger, H. Ejima, F. Caruso, Innovation in layer-by-layer assembly. Chem. Rev. 116 (2016) 14828-14867.

[3] C.D. Bain, E.B. Troughton, Y.-T. Tao, J. Eval, G.M. Whitesides, R.G. Nuzzo, Formation of monolayer films by the spontaneous assembly of organic thiols from solution onto gold. J. Amer. Chem. Soc. 111 (1989) 321-335.

[4] C. Creton, S. Gorb, Sticky feet: From animals to materials. MRS Bull. 32 (2007) 466-472.

[5] J. Ritu, S. Wairkar, Recent developments and clinical applications of surgical glues: An overview. Int. J. Biol. Macromol. 137 (2019) 95-106.

[6] H. Cho, G. Wu, J.C._Jolly, N. Fortoul, Z. He, Y. Gao, A. Jagota, S. Yang, Intrinsically reversible superglues via shape adaptation inspired by snail epiphragm. Proc. Natl. Acad. Sci. USA 116 (2019) 13774-13779. 
[7] T.S Sileika, G. Barrett, R. Zhang, K.H.A. Lau, P.B. Messersmith, Colorless multifunctional coatings inspired by polyphenols found in tea, chocolate and wine. Angewandte Chemie, 52 (2013) 10766-10770.

[8] H. Lee, S.M. Dellatore, W.M. Miller, P.B. Messersmith, Mussel-inspired surface chemistry for multifunctional coatings. Science, 318 (2007) 426-430.

[9] S.M; Kang, J. Rho, I.S. Choi, P.B. Messersmith, H. Lee, Norepinephrine: Material-independent, multifunctional, surface modification reagent. J. Amer. Chem. Soc. 131 (2009) 13224-13225.

[10] B. Shen, B. Xiang, H. Wu, Convenient surface functionalization of whole-Teflon chips with polydopamine coating. Biomicrofluidics, 9 (2015) art. 044111.

[11] I. Talon, A. Schneider, E. Mathieu, B. Senger, B. Frisch, C. Seguin, V. Ball, J. Hemmerlé, How polydopamine modulates biological responses to PTFE prostheses. Mater. Sci. Appl. 10 (2019) 377-392.

[12] Y. B. Lee, Y. M. Shin, J-H. Lee, I. Jun, J. K. Kang, J-C. Park, H. Shin, Polydopamine-mediated immobilization of multiple bioactive molecules for the development of functional vascular graft materials. Biomaterials, 33 (2012) 83438352.

[13] H. Lee, J. Rho, P.B. Messersmith, Facile conjugation of biomolecules onto surfaces via mussel adhesive protein inspired coatings, Adv. Mater. 21 (2009) 431435.

[14] H. Hu, B. Yu, Q. Ye, Y. Gu, F. Zhou, Modification of carbon nanotubes with nanothin polydopamine layer and polydimethylamino-ethylmeythacrylate brushes. Carbon 48 (2010) 2347-2353.

[15] H. Lee, N.F. Scherer, P.B. Messersmith, Single-molecule mechanics of mussel adhesion. Proc. Natl. Acad. Sci. USA 103 (2006) 12999-13003.

[16] B.P. Lee, P.B. Messersmith, J.N. Israelachvili, J.H. Waite, Mussel Inspired Adhesives and Coatings. Ann. Rev. Mater. Sci. 41 (2011) 99-132.

[17] N.F Della Vecchia, R. Avolio, M. Alfé, M.E. Errico, A. Napolitano, M. d'Ischia, Building-block diversity in polydopamine underpins a multifunctional eumelanin-type platform tunable through a quinone control point. Adv. Funct. Mater., 23 (2013) 1331-1340.

[18] M. lacomino, J.L. Paez, R. Avolio, A. Carpentieri, L. Panzella, G. Falco, E. Pizzo, M.E. Errico, A. Napolitano, A. del Campo, M. d'Ischia. Multifunctional Thin 
Films and Coatings from Caffeic Acid and a Cross-Linking Diamine. Langmuir 33 (2017) 2096-2102.

[19] B. Liu, L. Burdine, T. Kodadek, Chemistry of Periodate-Mediated Cross-Linking of 3,4-Dihydroxyphenylalanine-Containing Molecules to Proteins. J. Amer. Chem. Soc. 2006, 128 (2006) 15228-15235.

[20] Y. Liu, K. Ai, L. Lu, Polydopamine and its derivative materials: synthesis and promising applications in energy, environmental, and biomedical fields. Chem. Rev. 114 (2014) 5057-5115.

[21] V. Ball, D._Del Frari, M. Michel, J. Gracio, J.; M.J. Buehler, M. Singh, V. Toniazzo, D. Ruch, Deposition mechanism and properties of thin polydopamine films for high added value applications in surface science at the nanoscale. BioNanoScience. 2 (2012) 16-34.

[22] J. Liebscher, R. Mrowczynski, H.A. Scheidt, C. Filip, N.D. Hadade, R. Turcu, A. Bende, S. Beck,_Structure of Polydopamine: A Never-Ending Story? Langmuir 29 (2013) 10539-10548.

[23] D.R.Dreyer, D.J. Miller, B.D. Freeman, D.R. Paul, C.W. Bielawski, Perspectives on polydopamine. Chem. Sci. 4 (2013) 3796-3802.

[24] S. Hong, Y.S. Na, S. Choi, I.T. Song, W.Y. Kim, H. Lee, Non-covalent selfassembly and covalent polymerization co-contribute to polydopamine formation. Adv. Funct. Mater. 22 (2012), 4711-4717.

[25] S. Hong, Y. Wang, S.Y. Park, H. Lee, Fuzzy progressive cation-m assembly of biological catecholamines. Sci. Adv. 4 (2018) art eaat7457.

[26] V. Ball, Polydopamine Nanomaterials: Recent Advances in Synthesis Methods and Applications. Frontiers Bioengi. \& Biotechnol., 6 (2018) art. 109.

[27] N.C Holcomb, R-M. Bautista, S.G. Jarrett, K.M. Carter, M. Krentz Gober, J. A. D'Orazio., cAMP-mediated regulation of melanocyte genomic instability: a melanoma-preventive strategy. Adv. Protein Chem. \& Struct. Biol., 115 (2019) 247295.

[28] P. Meredith, T. Sarna, The Physical and Chemical Properties of Eumelanin. Pigment Cell Res., 19 (2006) 572-594.

[29] M. d'Ischia, K. Wakamatsu, A. Napolitano, S. Briganti, J.-C. Garcia-Borron, D. Kovacs, P. Meredith, A. Pezzella, M. Picardo, T. Sarna, J.D. Simon, S. Ito, Melanins and melanogenesis: methods, standards, protocols. Pig. Cell Melanoma Res., 26 (2013) 616-633. 
[30] J.D. Simon, D.N. Peles, The red and the black. Acc Chem Res. 2010, 43, $1452-$ 1460.

[31] J.J. Riez, J.B. Gilmore, R.H. McKenzie, B.J. Powell, M.R. Pederson, P. Meredith, Transition dipole strength of eumelanin. Phys. Rev. E., 76 (2007) art. 021915.

[32] V. Ball, Determination of the extinction coefficient of "polydopamine" films obtained by using $\mathrm{NaIO}_{4}$ as the oxidant Mater. Chem. Phys.,186 (2017) 546-551.

[33] F. Bernsmann, A. Ponche, C. Ringwald, J. Hemmerlé, J. Raya, B. Bechinger, J .-C. Voegel, P. Schaaf, V. Ball, Characterization of dopamine-melanin growth on silicon oxide. J. Phys. Chem. C, 113 (2009) 8234-8242.

[34] P. Meredith, J. Riesz, Radiative relaxation quantum yields for synthetic eumelanin. Photochem. Photobiol. 79 (2004) 211-216.

[35] C.T Chen, Eumelanin and polydopamine: self-assembly, structure and properties, PhD thesis, Massachusetts Institute of Technology, 2016.

[36] C.T Chen, J. Buehler, Polydopamine and eumelanin in various oxidation states. Phys. Chem. Chem. Phys., 20 (2018) 28135-28143.

[37] E. Kaxiras, A. Tsolakidis, G. Zonios, S. Meng, Structural model of eumelanin. Phys. Rev. Lett. 97 (2006) art.218102.

[38] C.T. Chen, V. Ball, J.J Almeida Gracio, M.K. Singh, V. Toniazzo, D. Ruch, M.J. Buehler, Self-assembly of tetramers of 5,6-dihydroxyindole is sufficient to explain the main physical properties of eumelanin. ACS Nano. 7 (2013) 1524-1532.

[39] Alfieri, M.L., R. Micillo, L. Panzella, O. Crescenzi, S.L. Oscurato, P. Maddalena, A. Napolitano, V. Ball, M. d'Ischia, Structural basis of polydopamine film formation: probing 5,6-dihydroxyindole-based eumelanin type units and the porphyrin issue. Appl. Mater. Interf. 10 (2018) 7670-7680.

[40] C.M.R. Clancy, J.D. Simon, Ultrastructural organization of eumelanin from Sepia Officinalis measured by atomic force microscopy, Biochemistry 40 (2001) 1335313360.

[41] M.G. Bridelli, Self-assembly of melanin studied by laser light scattering. Biophys. Chem. 73 (1998) 227-239.

[42] V. Ball, Impedance spectroscopy and zeta potential titration of dopa-melanin films produced by oxidation of dopamine. Colloids \& Surf. A. Phys. Chem Eng. Asp. 363 (2010) 92-97. 
[43] E.S. Bronze-Uhle, J.V. Paulin, M. Piacenti-Silva, C. Battocchio, M.LM. Rocco, C.F.D. Graeff, Melanin synthesis under oxygen pressure. Polym. Int. 65 (2016) 1339-1346.

[44] K-Y. Ju, Y. Lee, S.B. Park, J.-K. Lee, Bioinspired polymerization of dopamine to generate melanin-like nanoparticles having an excellent free-radical-scavenging property. Biomacromolecules 12 (2011) 625-632.

[45] M. Arzillo, G. Mangiapia, A. Pezzella, R.K. Heenan, A. Radulescu, L. Paduano, M. d'Ischia, Eumelanin buildup on the nanoscale: Aggregate growth/assembly and visible absorption development in biomimetic 5,6-dihydroxyindole polymerization. Biomacromolecules, 13 (2012) 2379-2390.

[46] F. Ponzio, P. Bertani, V. Ball, Role of surfactants in the control of dopamineeumelanin particle size and in the inhibition of film deposition at solid-liquid interfaces. J. Colloid Interface Sci. 431 (201) 176-179.

[47] A. Schneider, J. Hemmerlé, M. Allais, J. Didierjean, M. Michel, M. d'Ischia, V. Ball, Boric acid as an efficient agent for the control of PDA self-assembly and surface properties, ACS Applied materials interfaces, 10 (2017) 7574-7580.

[48] M. Salomäki, T. Ouvinen, L. Marttila, H. Kivelä, J. Leiro, E. Mäkilä, J. Lukkari, Polydopamine nanoparticles prepared using redox active transition metals, J. Phys. Chem. B, 2019, 123 (2019) 2513-2524.

[49] F. Ponzio, J. Barthès, J. Bour, M. Michel, P. Bertani, J. Hemmerlé, M. d'Ischia, V. Ball, Oxidant Control of polydopamine surface chemistry in acids: a mechanismbased entry to superhydrophilic-superhydrophobic coatings, Chem. Mater., 28 (2016) 4697-4705.

[50] X. Wang, Z. Chen, P. Yang, J. Hu, Z. Wang, Y. Li, Size control of melanin-like polydopamine nanoparticles by tuning radicals, Polym. Chem., 10 (2019) 4194-4200. [51] F. Liu, X. He, J. Zhang, H. Chen, H. Zhang, Z. Wang, Controllable synthesis of polydopamine nanoparticles in microemulsions with $\mathrm{pH}$-activatable properties for cancer detection and treatment, J. Mater. Chem. B, 2015, 3 (2015) 6731-6739.

[52] N.F. della Vecchia, P. Cerruti, G. Gentile, M.E. Errico, V. Ambrogi, G. D'Errico, S. Longobardi, A. Napolitano, L. Paduano, C. Carfagna, d'Ischia,_Artificial Biomelanin: Highly Light-Absorbing Nano-Sized Eumelanin by Biomimetic Synthesis in Chicken Egg White. Biomacromolecules 15 (2014) 3811-3816. 
[53] O.I. Strube, A. Büngeler, W. Bremser, Site-specific in situ synthesis of eumelanin nanoparticles by an enzymatic autodeposition-like process. Biomacromolecules 16 (2015) 1608-1613.

[54] O.I. Strube, A. Büngeler, W. Bremser, Enzyme-mediated in situ synthesis and deposition of nonaggregated melanin protoparticles. Macromol. Mater. \& Eng. 301 (2016) 801-804.

[55] C. Bergtold, D. Hauser, A. Chaumont, S. El Yakhlifi, M. Mateescu, F. Meyer, M.H. Metz-Boutigue, B. Frisch, P. Schaaf, D. Ihiawakrim, O. Ersen, C.A. Monnier, A.Petri-Fink, B. Rothen-Rutishauser, V. Ball, Mimicking the chemistry of natural eumelanin synthesis: the KE sequence in polypeptides and in proteins allows for a specific control of nanosized functional polydopamine formation, Biomacromolecules, 19 (2018) 3693-3704.

[56] A. Chassepot, V. Ball, Human serum albumin and other proteins as templating agents for the synthesis of nanosized dopamine-eumelanin. J. Coll. Interf. Sci., 414 (2014) 97-102.

[57] S. El Yakhlifi, D. Ihiawakrim, O. Ersen, V. Ball, Enzymatically Active Polydopamine @ Alkaline Phosphatase Nanoparticles Produced by $\mathrm{NaIO}_{4}$ Oxidation of Dopamine, Biomimetics, 3 (2018) art. 3693.

[58] A. Lampel, S.A. McPhee, H-A. Park, G.G. Scott, S. Humagain, D.R. Hekstra, B. Hoo, P.W.J.M. Frederix, T.-D. Li, R.R. Abzalimov, S.G. Greenbaum, T. Tuttle, C. Hu, C.J. Bettinger, R.V. Ulijn, Polymeric peptide pigments with sequence-encoded properties, Science, 356 (2017) 1064-1068.

[59] X. Yu, H. Fan, L. Wang, Z. Jin, Formation of polydopamine nanofibers with the aid of folic acid. Angew Chem. Int. Ed. 53 (2014) 12600-12604.

[60] H. Fan, X. Yu, Y. Liu, Z. Shi, H. Liu, Z. Nie, D. Wu, Z. Jin, Folic-acid polydopamine nanofibers show enhanced ordered-stacking via $\pi-\pi$ interactions. Soft Matter, 11 (2015) 4621-4629.

[61] D.R. Amin, C.J. Higginson, A.B. Korpusik, A.R. Gonthier, P.B. Messersmith, Untemplated resveratrol-mediated polydopamine nanocapsule formation. ACS Appl. Mater. Interf. 10 (2018) 34792-34801.

[62] A. Postma, Y. Yan, Y. Wang, A.N. Zelikin, E. Tjipto, F. Caruso, Selfpolymerization as a versatile and robust technique to prepare polymer capsules. Chem; Mater. 21 (2009) 3042-3044. 
[63] L. Zhang, J. Shi, Z. Jiang, Y. Jiang, S. Qiao, J. Li, R. Wang, R. Meng, Y. Zhu, Y. Zheng, Bioinspired preparation of polydopamine microcapsule for multienzyme system construction. Green Chem. 13 (2011) 300-306.

[64] J. Xue, W. Zheng, L. Wang, Z. Jin, Scalable fabrication of polydopamine nanotubes based on curcumin crystals. ACS Biomater. Sci. \& Eng. 2 (2016) 489493.

[65] D. Yan,, P.C. Xu, Q. Xiang, H.R. Mou, J.Q. Xu, W.J. Wen, X.X. Li, Y. Zhang, Polydopamine nanotubes: bio-inspired synthesis, formaldehyde sensing properties and thermodynamic investigation. J. Mater. Chem. A 4 (2016) 3487-3493.

[66] Y. Liu, K. Ai, J. Liu, M. Deng, Y. He, L. Lu, Dopamine-melanin colloidal nanospheres an efficient near-infrared photothermal therapeutic agent for in vivo cancer therapy. Adv. Mater. 25 (2013) 1353-1359.

[67] D. Hauser, M. Estermann, A. Milosevic, L. Steinmetz, D. Vanhecke, D. Septiadi, B. Drasler, A. Petri-Fink,_V. Ball, B. Rothen-Rutishauser, Polydopamine/Transferrin Hybrid Nanoparticles for Targeted Cell-Killing, Nanomaterials, 8 (2018) art. 1065.

[68] X. Bao, J. Zhao, J. Sun, M. Hu, X. Yang, Polydopamine nanoparticles as efficient scavengers for reactive oxygen species in periodontal disease, ACS Nano, 2018, 12, 9, 8882-8892.

[69] Y. Huang, Y. Li, Z. Hu, X. Yue, M.T. Proetto, Y. Jones, N.C Gianneschi, Mimicking Melanosomes: polydopamine nanoparticles as artificial microparasols, ACS Central Science, 2017, 3(6), 564-569.

[70] H. Ma, Y. He, H. Liu, L. Xu, J. Li, M. Huang, Y. Wei, Anchoring of Prussian Blue nanoparticles on polydopamine nanospheres as an efficient peroxidase mimetic for colorimetric sensing, Colloids and Surfaces A, 577(2019) 622-629.

[71] C. Lu, F. Sun, Y. Liu, Y. Xiao, Y. Qlu, H. Mu, J. Duan, Versatile Chlorin e6based magnetic polydopamine nanoparticles for effectively capturing and killing MRSA, Carbohydrate. Polym., 218 (2019) 289-298.

[72] B. Poinard, S. Zhan Yuan Neo, E. Li Ling Yeo, H. Peng Sin Heng, K. G Neoh, J. Chen Yong Kah, Polydopamine nanoparticles enhance drug release for combined photodynamic and photothermal therapy. ACS Appl. Mater Interf., 10 (2018) 2112521136. 
[73] H. Xiong, J. Xu, C. Yuan, X. Wang, W. Wen, X. Zhang, S. Wang, Oxydation controlled synthesis of fluorescent polydopamine for the detection of metal ions., Microchem. J., 2019, 147, 176-182.

[74] Z. Wang, C. Xu, Y. Lu, G. Wei, G. Ye, T. Sun, J. Chen, Microplasma electrochemistry controlled rapid preparation of fluorescent polydopamine nanoparticles and their application in uranium detection. Chem. Eng. J. 344 (2018) 480-486.

[75] K. Qu, Y. Wang, A. Vasileff, Y. Jiao, H. Chen, Y. Zheng, Polydopamine-inspired nanomaterials for energy conversion and storage. J. Mater. Chem. A. 6 (2018) 21827-21846.

[76] M. Xiao, Y;-e. Li, M.C. Allen, D.D. Deheyn, X. Yue, J. Zhao, N.C. Gianneschi, M. D. Shawkey, A. Dhinojwala, Bio-structural colors produced via self-assembly of synthetic melanin nanoparticles. ACS Nano (2015) 5454-5460.

[77] A. Kawamura, M. Kohri, S. Yoshioka, T. Taniguchi, K. Kishikawa, Structural color tuning: mixing melanin-like particles with different diameters to create neutral colors. Langmuir 33 (2017) 3824-3830.

[78] T. Iwasaki,Y. Tamai, M. Yamamoto, T. Taniguchi, K. Kishikawa, M. Kohri, Melanin precursor influence on structural colors from artificial melanin particles: polyDOPA, polydopamine and polynorepinephrine. Langmuir 34 (2018) 1181411821.

[79] M. Kohri, K. Uradokoro, Y. Nannichi, A. Kawamura, T. Taniguchi, K. Kishikawa, Hairy polydopamine particles as platforms for photonic and magnetic materials. Photonics 5 (2018) art. 36.

[80] J. Zi, X. Yu, Y. Li, X. Hu, C. Xu, X. Wang, X. Liu, R. Fu, Coloration strategies in peacock feathers. Proc. Natl. Acad. Sci. USA 100 (2003) 12576-12578.

[81] A. A. Kumar, S.D. Bhagat, R. Ramakrishnan, A. Srivastava, Chirally twisted ultrathin polydopamine nanoribbons: synthesis and spontaneous assembly of silver nanoparticles on them. Chem. Eur. J. 25 (2019) 12905-12910. 